\title{
Dual type (Type I, Type IIIa) ileal atresia with minor omphalocele
}

\author{
Nasser Shukri*, Hanan Taher, Ahmad Al Shabasi, Omar Al Matari, Ohoud Al Amoudi, Rizq Al Amri and Aml Sallam* \\ General East Jeddah Hospital, KSA
}

\begin{abstract}
Omphaolcele minor is a congenital midline umbilcal defect of less than $5 \mathrm{~cm}$ in diameter that results in herniation of the intra -abdominal contents covered by a lining of peritonium and amnion. We report a rare case associated with ileal atresia having dual different types.
\end{abstract}

\section{Case report}

A 38 years old multiparous admitted and delivered by caeserian section at 38 weeks of gestation, only on the $37^{\text {th }}$ week prenatal ultrasound showed suspicious presence of omphalocele. Baby was born full term weighing $3.2 \mathrm{kgs}$ with apgar scores of 9 to 10 at 1 to 5 minutes respectively.

Physical examination showed a minor omphalocele with $3 \mathrm{~cm}$ defect. Baby had no associated anomalies including congenital heart, chromosomal, or renal genitourinary or facial or skeletal anomalies.

Operation performed following day, findings in the sac was only part of small intestine but complete abdominal exploration showed presence of type I ileal atresia $4 \mathrm{~cm}$ proximally from the terminal ileum (Figure 1). Then after careful dissection of nearby adhesions distally type IIIa ileal atresia was found (Figure 2). End to side ileocolic anastomosis was done with resection of the most of the proximal dilated part. Postoperative follow up was uneventful and baby was discharged after 10 days.

\section{Discussion}

An omphalocele represents an embryological defect of the umbilical ring and medial segments of the two lateral abdominal folds during fetal growth and can be divided into two groups depending on the size of the hernial defect [1]. Omphalocele defects vary in size, with $5 \mathrm{~cm}$ diameter in minor to $>5 \mathrm{~cm}$. The incidence is nearly 2.5 cases per 10,000 live births. Most 'minor' cases have diameters less than $5 \mathrm{~cm}$ and contain mostly loops of small bowel. Isolated minor omphaloceles with no associated structural and chromosomal anomalies are known to have a favorable prognosis [2]. Immediate repair of omphalocele minor is the recommended treatment, the contents are usually adherent to the sac especially for patients who have irreducible contents, ruptured sac.

The rates of morbidity and mortality are related to the intestinal anomalies and not to cardiac defects associated mainly with omphalocle major [3]. The association of the intestinal atresia and omphalocele minor is rare (Figure 3). Although intestinal atresia maybe due to genetic causes, Intrauterine mesenteric vascular accidents, and of unknown origin recent several literatures supports that the development of intestinal atresia in omphalocele requires a small abdominal wall defect

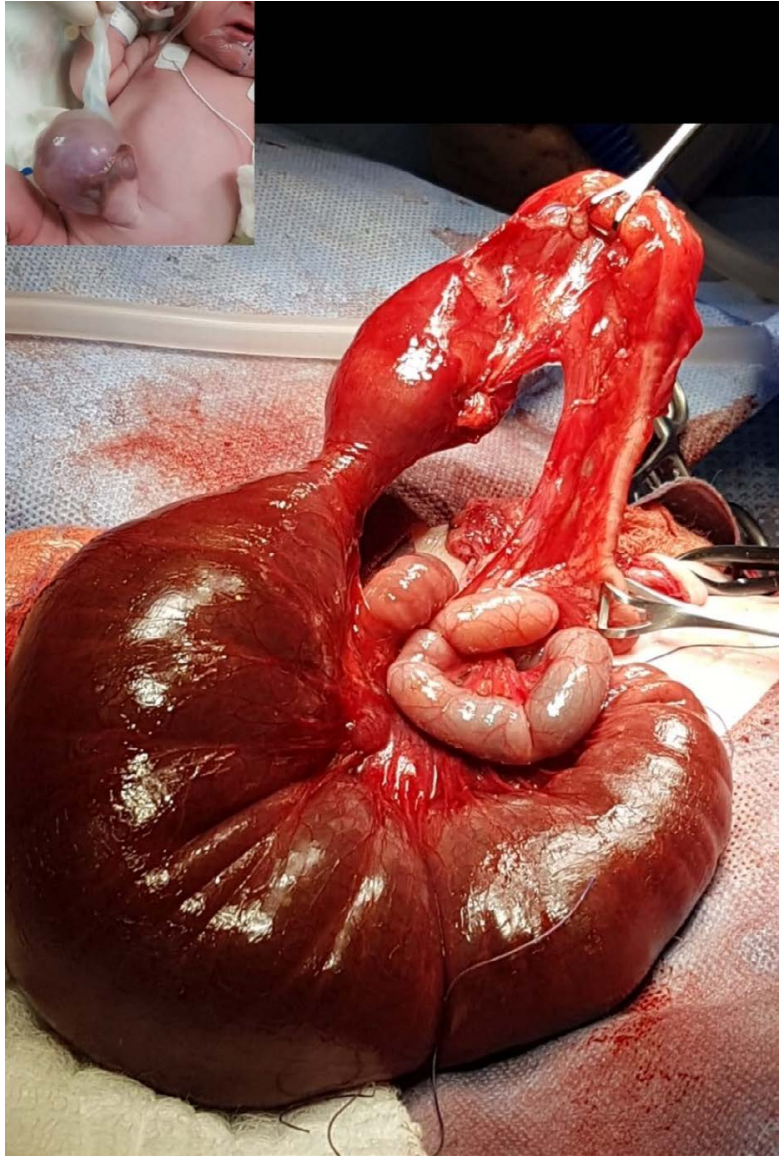

Figure 1. Type I ileal atresia $4 \mathrm{~cm}$ proximally from the terminal ileum

*Correspondence to: Nasser Shukri, General East Jeddah Hospital, KSA, E-mail: nas007_sa@hotmail.com

Key words: ileal atresia, omphalocele

Received: November 14, 2020; Accepted: November 23, 2020; Published: November 30, 2020 


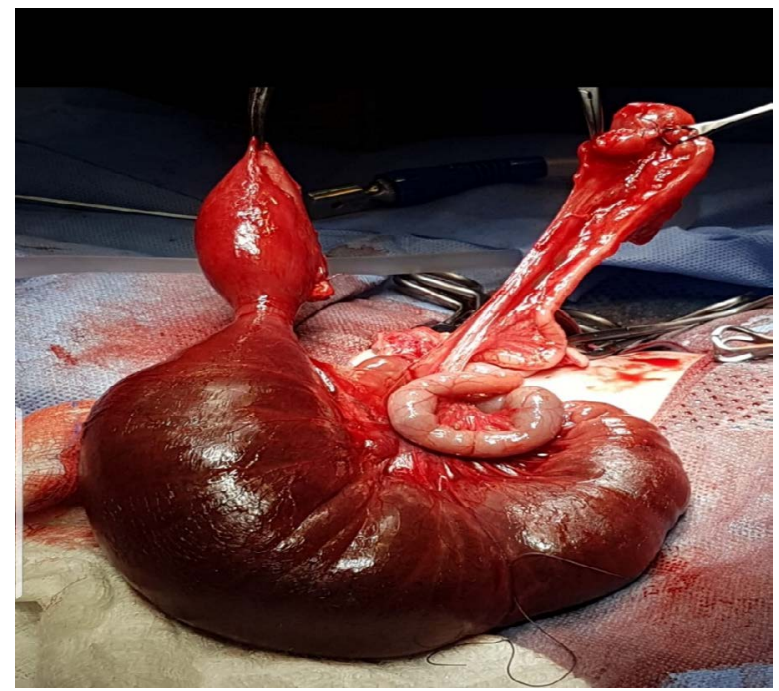

Figure 2. Type IIIa ileal atresia
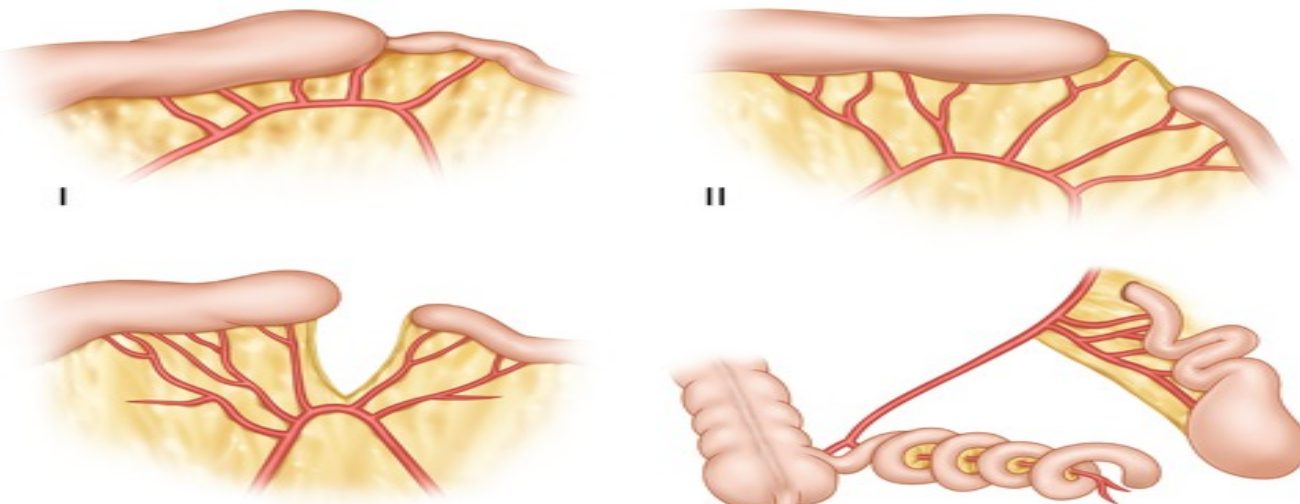

IIIa
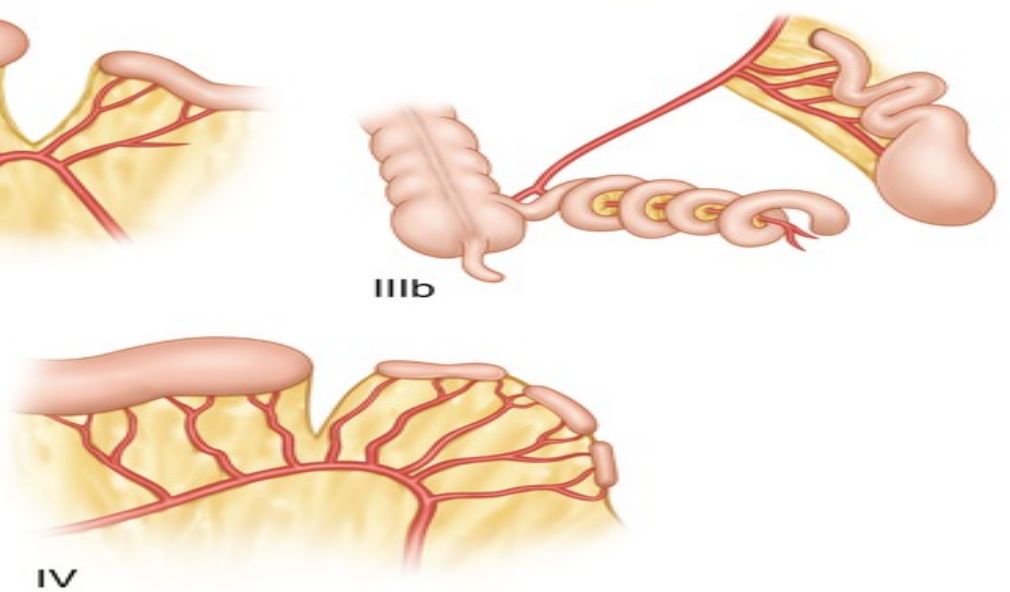

Figure 3. Types of intestinal atresia

capable of intestinal and mesenteric compression by the interruption of blood supply resulting in ischemia [4,5]. Pratap et al. [6] described a case of ileum entrapped within the omphalic ring and that entrapped segment of ileum was atretic. Patel et al. [6] reported an unusual case of ileal atresia resulting from antenatal strangulation of Meckels diverticulum in omphalocele minor. The first association of ileal atresia with Omphalocele minor is the single isolated type. Salomon [7] reported an ileal atresia in a case report and Wakhlu [8] reported only one ileal atresia, in a serie of 45 cases with minor omphaloceles. The second association is multiple atresia of same type being associated with minor omphaloceles in which Cortese et al. [9] reported one case of multiple intestinal atresia with omphalocele and recently Etensel [10] report a new association of a minor omphalocele, type IV ileal atresia and congenital ostomy. No report has been described in the literature of association with different dual types lleal Atresia (Type1 Type Illa) in the same patient. Our case emphasizes the occurrence of this very rare combination of congenital malformation.

\section{Conclusions}

Although this is the first report of omphalocele minor described in the literature to be associated with different types of ileal atresia in a patient, much attention should be paid to early timing of operation not only due to possible intestinal compression by a tight defect but also the recognition of undetected intestinal anomalies during the surgical correction.

\section{Acknowledgments}

Special thanks to the head of department of histopathology Dr Abdul Wahid Hamadaleel for his guidance and help. OR technician $\mathrm{Mr}$ 
Abd Rahman Bin Besher and OR nurses Mr Mansour AI-Mahzari and Miss Rowena Puna for their kind assistance in the operating theatre. Neonatal intensive care unit Dr Awatif Suliman and nurses Miss Sabah Ismail and Miss Moudi AI-Dosseri for their persistent dedicated care for the patient.

\section{References}

1. Cooney DR (1998) Defects of the abdominal wall. In Pediatric surgery (5th edn), Mosby, Toronto

2. Kumar HR, Jester AL, Ladd AP (2008) Impact of omphalocele size on associated conditions. J Pediatr Surg 43: 2216-2219 [Crossref]

3. Raashid Hamid, Gowhar Mufti, Sajad A Wani, Imran Ali, Bhat NA, et al. (2015) Importance of the Early Management of Omphalocele Minor. J Neonatal Biol 4: 2.

4. Clogg HS (1904) Congenital intestinal atresia. Lancet 2: 1770-1774.
5. Pratap A, Yadav RP, Bajracharya A, Agrawal A, Bhatta N, et al. (2008) Intrauterine omphalic ileal entrapment as an unusual cause of ileal atresia: report of a case. Surg Today 38: 141-143. [Crossref]

6. Patel RV, Khoo AK, De Coppi P, Pierro A (2013) Ileal atresia secondary to antenatal strangulation of Littre's hernia in an exomphalos minor. BMJ Case Rep 10: 1136 [Crossref]

7. Salomon LJ, Benachi A, Auber F, Bonnard A, Nihoul-Fékété C, et al. (2002) Omphalocele: beyond the size issue. J Pediatr Surg 37: 1504-1505.

8. Wakhlu A, Wakhlu AK (2000) The management of exomphalos. J Pediatr Surg 35 73-76. [Crossref]

9. Cortese MG, Morra I, Marchese C, Costan Tino S, Forni M, et al. (2001) Association between multiple intestinal atresia and omphalocele: A case report. Pediatr Pathol Mol Med 20: 203-207. [Crossref]

10. Barlas E, Ali O, Özge Ç, İbrahim M, Münevver K, et al. (2014) Minor omphalocele, type IV Ileal atresia, congenital ostomy. Open Journal of Pediatrics 4: 177-179

Copyright: $\odot 2020$ Shukri N. This is an open-access article distributed under the terms of the Creative Commons Attribution License, which permits unrestricted use, distribution, and reproduction in any medium, provided the original author and source are credited. 